\title{
An Algorithm of Filtering InSAR Interferogram Based on the Combination of Wavelet Transform and Sigma Filter
}

\author{
Cai Guolin, Zhang Aoli, Feng Dejun, Sun Meiling \\ (Dept. of RS and GIS, Southwest Jiaotong University, \\ Chengdu 610031, China)
}

\begin{abstract}
In order to enhance the quality in filtering InSAR interferograms, this paper proposes and constructs a Wavelet-Sigma filter (WSF) in view of the respective merits of wavelet transform and Sigma filter. The WSF and its computer program are developed to remove interferogram noise. To validate the proposed filter, the localized area around Bam of Iran is selected as the testing site an ASAR C-band interferogram is utilized as the source data for filtering. Several indicators, including visualization effect, standard deviation of phase derivatives and number of residues, are taken into account to assess the effectiveness of this filter. The results show that WSF has some prominent advantages in terms of denoising and edge protection, if compared with two typical methods presented previously, i.e., wavelet soft-threshold filter and Sigma filter.
\end{abstract}

Key words: InSAR Interferogram, Wavelet-Sigma filter, Residues

\section{I . INTRODUCTION}

The main data processing steps of Synthetic Aperture Radar Interferometry (InSAR) include: SAR image co-registration, interferogram production, reference plane phase remove, interferogram filter, terrain influence remove, phase unwrapping, geometric transformation, and etc. The key step is phase unwrapping. However, due to the effects of several factors, i.e., thermal noise, temporal and geometrical decorrelation as well as error of image co-registration, the interferogram computed from a pair of SAR images (acquired over the same region) has large numbers of residues (noises). The noises increase difficulty in phase unwrapping, and even result in failure of phase unwrapping ${ }^{[1]}$. Therefore, before phase unwrapping, it is necessary to carry on interferogram filtering to reduce the noises, which can ensure accuracy and reliability of unwrapped phase data.

Various filters have been proposed to reduce interferogram noises, such as median filter, mean filter ${ }^{[2]}$, Goldstein filter ${ }^{[3]}$, adaptive filter ${ }^{[4]}$, wavelet filter ${ }^{[5-6]}$ and etc. However, there are many disadvantages in the above filters: median filter works well when the current pixel is noise. Otherwise, when it is not noise or median, this filter will fail. Mean filter prevails in ESA and JPL, but it is apt to blur interferogram fringe. Goldstein filter is widely used now, with good performance in high SNR but dissatisfied performance in low SNR. Adaptive filter, particularly local adaptive filter, makes algorithms complicated and time-consuming, with weak stability and operability which results from the tasks of slope degree calculating, interferogram fringe positioning, fringe

Fund Projects: The work of this paper is supported by National Basic Research Program of China (973) (No. 2012CB719901), National Natural Science Fund Item (No. 41074005, 41271448, 41201434), the Fundamental Research Funds for the Central Universities (No. 2682013CX009, SWJTU09ZT01, SWJTU10ZT02, SWJTU11CX139, SWJTU11ZT13, SWJTU09BR015), Item of China Ministry of Education (No. 12YJAZH124).

\author{
Zhuo Song \\ (Sichuan Remote Sensing Geomatics Institute, Chengdu \\ 610100, China)
}

orientation deciding and complex phase unwrapping. In addition, all these algorithms have some contradiction between noise suppression and accuracy maintenance. Wavelet filter has been a hot research topic in recent years, and a number of investigators have already introduced it into filtering InSAR interferograms ${ }^{[5-6]}$. However, these filters have good effects in theory, but not in practice, and the denoising effects highly depend on the determination of threshold values. In view of the disadvantages of a single filter, many investigators ${ }^{[5,7]}$ have proposed to combine the merits of multiple filters to carry on filtering. These algorithms have showed great potential in filtering the conventional images ${ }^{[5]}$. In the aspect of InSAR interferogram, few scholars ${ }^{[5,7]}$ have taken the lead in carrying on the beneficial research.

In order to conduct a further study of multi-filters combinational algorithm and raise SNR of interferograms, this paper proposes an algorithm of Wavelet-Sigma combined (WSC) filter, and discusses how to filter noises in the interferograms by utilizing the merits of Wavelet transform and Sigma filter. To validate the proposed filter, one ASAR C-band interferogram over Bam, Iran is selected to carry on the experiment of filter and analysis, and the result will be compared with the results of two typical filters, i.e., Wavelet soft-threshold filter and Sigma filter.

\section{WAVELET-SIGMA COMBINED (WSC) FILTER FOR INTERFEROGRAM}

\section{A. Wavelet Filter Based on Multi-resolution Analysis}

An InSAR interferogram could be considered as a complex matrix, if using the Mallat algorithm to decompose and reconstruct the interferogram in multiple scales, we need to obtain real matrix and imaginary matrix of the interferogram first. Assuming that $f(x, y)$ represents real matrix or imaginary matrix of the interferogram, its two dimension wavelet transform is defined as following:

$$
W_{j}^{\lambda} f(x, y)=f^{*} \psi_{j}^{\lambda}(x, y)=\iint_{R^{2}} f(u, v) \psi_{j}^{\lambda}(x-u, y-v) d u d v
$$

Where: $j$ is decomposing scale, $\lambda$ are three low frequency components, $R^{2}$ is the collectivity of square integrable function in real number set, $\psi$ is wavelet function.

Assuming that $H$ and $G$ are corresponding filter coefficient matrixes of scale function $\varphi(x)$ and $\psi(y)$, its two dimension wavelet can be decomposed as following: 


$$
\left\{\begin{array}{l}
C_{j+1}=H C_{j} H^{*} \\
D_{j+1}^{h}=G C_{j} H^{*} \\
D_{j+1}^{v}=H C_{j} G^{*} \\
D_{j+1}^{v}=G C_{j} G^{*}
\end{array} \quad(j=0,1,2, \cdots, j-1)\right.
$$

Where: $h$ is horizontal coefficients, $v$ is vertical coefficients, and $d$ is diagonal coefficients. $H^{*}$ and $G^{*}$ are conjugate transpose matrixes of $H$ and $G$ respectively. Its corresponding reconstructing algorithm is as following:

$$
C_{j-1}=H^{*} C_{j} H+G^{*} D_{j}^{h} H+H^{*} D_{j}^{v} G+G^{*} D_{j}^{d} G
$$

Wavelet filter is composed of three steps: wavelet transform, wavelet coefficient nonlinear processing to filter noise and inverse wavelet transform. In the $2^{\text {nd }}$ step, filter processing is conducted in decomposed high frequency coefficients while low frequency ones are preserved. The reason is that there is lots of information contained in low frequency coefficients in wavelet decomposing, change of those will cause change of image. Meanwhile, some important wavelet coefficients with big absolute value are not noises but fringe signals which should be preserved too. Moreover, noises distribute in high frequency coefficients when firstly decomposed. Noises become fewer as the decomposing processing. It needs to be clarified that firstly wavelet filter is not to smooth but to remove all noises with all signals preserved, without thinking of their frequency range. Secondly, it processes wavelet coefficients in wavelet domain [9]

Compared with other general algorithms, the algorithm based on multi-resolution wavelet transform has following advantages: (1) The entropy of interferogram is decreased in wavelet domain due to the sparse distribution of wavelet coefficients. (2) Because of the method of multi-resolution, the algorithm of wavelet transform can describe the non-stationary characteristics of interferogram very well, i.e., edge and spike. So it avails the feature's extraction and protection. (3) Decorrelation of the interferogram can be achieved in wavelet domain, and the noises after Wavelet transform have the whitening trend. Therefore, denoising can be realized more easily in wavelet domain than time domain and space domain. Through the first and the second advantages, it can be concluded that wavelet filter is able to well preserve abrupt changes and fringes of image as removing noises ${ }^{[10]}$.

\section{B. Sigma Filter}

Sigma filter is Sigma probability based on Gaussian distribution with mean filter property ${ }^{[11]}$. The principles when it is used in interferogram are as following:

(1) Defining a window with size of $(2 n+1,2 m+1)$, moving it across interferogram real matrix and imaginary matrix and calculating its mean value $X_{\text {mean }}$ and standard deviation $\sigma$.
(2) Setting the window's upper limit threshold $X_{\max }$ and the lower limit threshold $X_{\min \text {. }}$

$$
\left\{\begin{array}{l}
X_{\text {max }}=X_{\text {mean }}+u \sigma \\
X_{\text {min }}=X_{\text {mean }}-u \sigma
\end{array} \quad(u=1,2,3)\right.
$$

(3) When moving the pixel value $X_{k, l}$ in this window, that is:

$$
\delta_{k, l}= \begin{cases}1 & X_{\min } \leq X_{k, l} \leq X_{\max } \\ 0 & \text { others }\end{cases}
$$

Where: $\delta_{k, 1}$ is eigenvalues of the pixel. After calculating using formula (5), we get the filtered value of the window's center point $X_{i, j}$.

$$
\begin{gathered}
S=\sum_{k=i-n}^{i+n} \sum_{l=j-m}^{j+m} \delta_{k, l} \\
\hat{X}_{i, j}=\sum_{k=i-n}^{i+n} \sum_{l=j-m}^{j+m} \delta_{k, l} X_{k, l} / S
\end{gathered}
$$

In order to smooth without isolated points, threshold $K$ is brought in. If the total number of the pixels within Sigma extent $S$ is smaller than or equal to $K$, the center pixel value is replaced with its 4-neighborhood pixels equal value (For window $7 \times 7, K$ is smaller than 4 . For window $5 \times 5, K$ is smaller than 3 ). Sigma filter could remove the influence on center pixel by pixels further than $u$ times standard deviation, which are often considered as noises, and preserve fringe information ${ }^{[11]}$.

\section{Structure and Filter Implementation of Wavelet-Sigma Filter}

Sigma filter is based on the statistical character of digital signal. It can eliminate noises but not that competent. It needs several times of processing, which may result in loss of image information ${ }^{[8]}$. Referring to chapter 2.1 and 2.2, wavelet transform algorithm and Sigma filter are very complementary, which means that we can perform filter by combining the advantages of wavelet transform, such as low entropy, multi-resolution, Gaussian noise whitening and decorrelation superiority, with the advantages of Sigma filter, such as non-stationary characteristics.

Thus, the paper attempts to combine wavelet transform and Sigma filter to eliminate InSAR interferogram noises. Firstly, decompose real matrix or imaginary matrix using wavelet multi-resolution analysis. Secondly, use Sigma filter to filter decomposed part of high frequency. Lastly, reconstruct the wavelet coefficients to get decomposed real matrix or imaginary matrix. The flow is shown as Fig.1 (LL, $\mathrm{HH}, \mathrm{VV}, \mathrm{DD}$ are low frequency coefficients, horizontal high frequency coefficients, vertical high frequency coefficients and diagonal high frequency coefficients).

Using the algorithm of WSC to filter an InSAR interferogram, the steps are as following:

(1) Extract real part and imaginary part from the 
interferogram (viz., signal $y$ with noise), and get two matrixes.

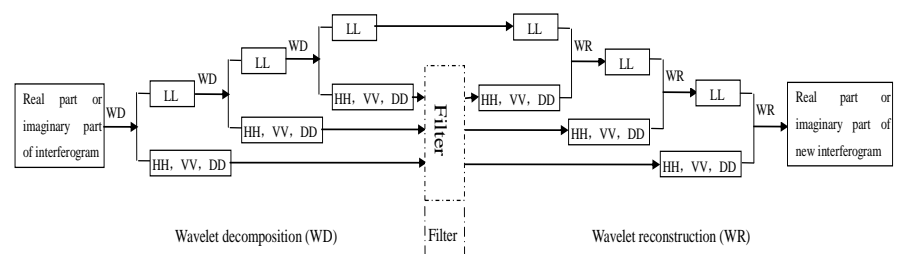

Fig.1 Flowchart of filtering based on Wavelet decomposition and reconstruction

(2) Decompose the two matrixes by use of Wavelet transform. This paper uses sym4 Wavelet to carry out three-layer (i.e., three-scale, $j=1,2,3$ ) decomposition.

(3) Define a Sigma filter window with the size of ${ }^{[6]}$

$$
w_{j}=w_{0} 2^{j-1}-1
$$

Where: $u=2, j \geqslant 2, w_{0}=5$ is the first layer window size, i.e. $n=m=2$. Use this filter window to filter wavelet coefficients in each decomposed scale.

(4) Perform wavelet reconstruction to get a new matrix, i.e. a new real matrix or a new imaginary matrix.

(5) Combine the new real matrix and the new imaginary matrix, and get a new interferogram.

\section{Filter experiment and analysis}

\section{A. Experiment Data and Filter Assessment Criterion}

According to the description in part 2, the corresponding Matlab programs of WSC filter is compiled. In order to validate the effects of WSC, two ASAR C-band SAR interferogram over part of Bam, Iran (wavelength is $5.6 \mathrm{~cm}$, images are got at 2003.6.11 and 2004.2.11 respectively) are selected to carry on the experiment. The image block size for wavelet analysis is $600 \times 800$ pixels

The experiment not only analyzes the filter effects of this algorithm, but also compares the result of WSC filter with the results of wavelet soft-threshold filter and Sigma filter to validate the efficiency and superiority of this algorithm. Regarding wavelet soft-threshold filter, signal is transformed to frequency domain at first, which can avoid loss of image characteristics information. While Sigma filter is based on the statistical character of digital signal, which can eliminate noises with fringe information preserved.

The appraisal parameters selected for the filter results of this experiments are following: visualization effect of image, chart of PDSD and histogram of PDSD ${ }^{[12]}$, number of residues ${ }^{[12]}$ (the less the residues are, the better the quality of the interferogram is). The PDSD is defined by the equation [12].

$$
z_{m, n}=\frac{\sqrt{\sum\left(\Delta x_{i, j}-\overline{\Delta x}_{m, n}\right)^{2}}+\sqrt{\sum\left(\Delta y_{i, j}-\overline{\Delta y}_{m, n}\right)^{2}}}{k^{2}}
$$

Where: the terms $\Delta x_{i, j}$ and $\Delta y_{i, j}$ represent gradient values in $x$ and $y$ direction; the terms $\overline{\Delta x}_{m, n}$ and $\overline{\Delta y}_{m, n}$ are the

averages gradient of $x$ and $y$ direction in $k \times k$ windows, the term $Z_{m, n}$ is the PDSD value of the center of window. PDSD is considered as the effective measure that is directly used to appraise the quality of interferogram ${ }^{[12]}$, the bigger its value is, the worse the quality of the interferogram is.

\section{B. Experiment Result and Contrastive Analysis}

Original interferogram over Bam, Iran is showed in fig. 2 (a). Fig.2 (b)-(d) separately demonstrates the results of wavelet soft-threshold filter, $5 \times 5$ Sigma filter and WSC filter that is proposed in this paper. Fig.3 (a)-(d) demonstrates the results of PDSD that correspond to fig.2 (a)-(d), and their values correspond to color bar (the bigger its value is, the worse the quality of the interferogram is). Fig.4 (a)-(d) demonstrates the histograms of PDSD that correspond to fig. 3 (a)-(d) (the smaller its value is, the better the quality of the interferogram is). The Stat. of residues for four interferograms of fig. 2 is listed in tab.1.

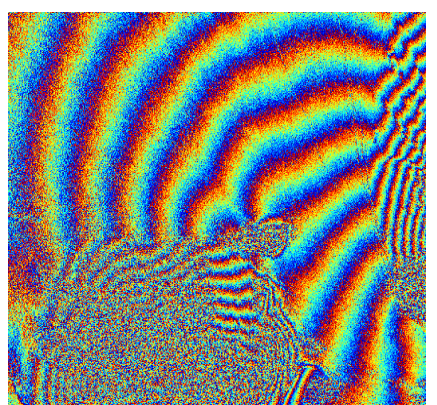

(a) an original interferogram

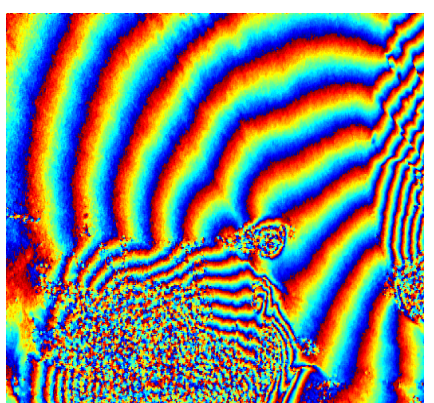

(c) an interferogram by Sigma filter

Fig.2 Original interferogram and its filtered versions by 3 methods over Bam

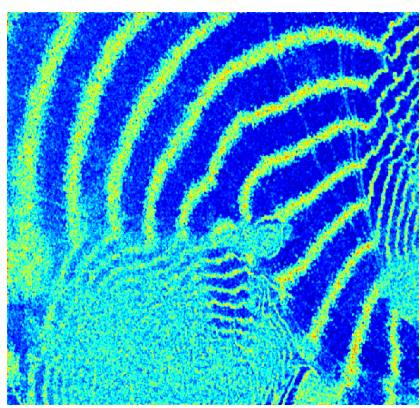

(a) original PDSD

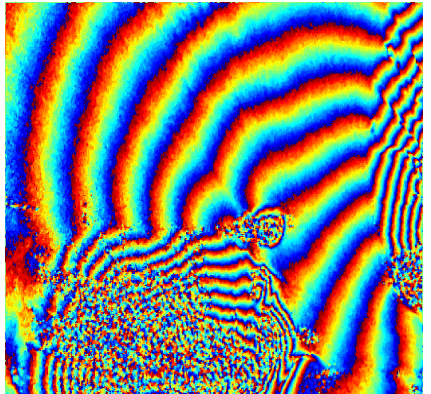

(b) an interferogram by wavelet soft-threshold filter

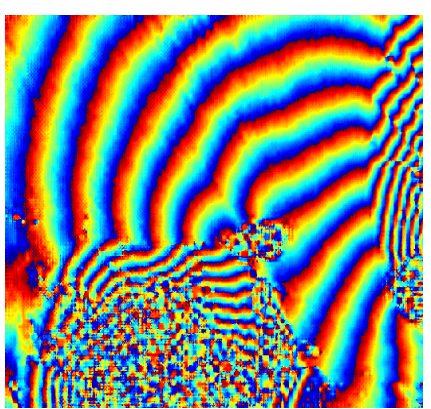

(d) an interferogram by WSC filter

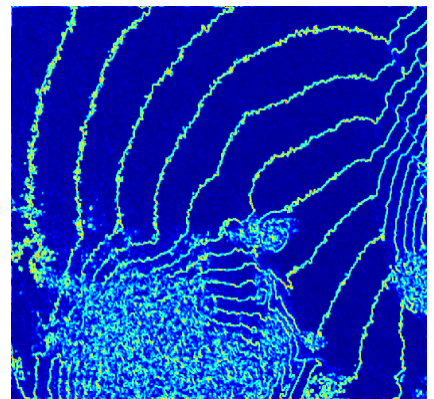

(b) PDSD by wavelet soft-threshold filter 


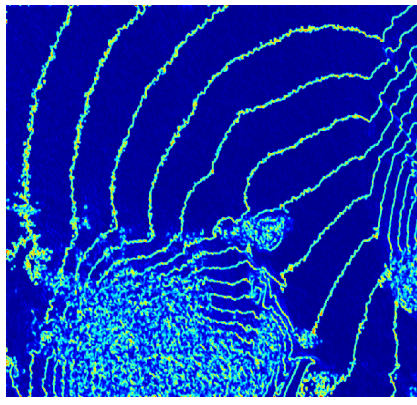

(c) PDSD by Sigma filter

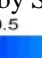

Fig.3 Comparison of phase-derivative standard deviation over Bam

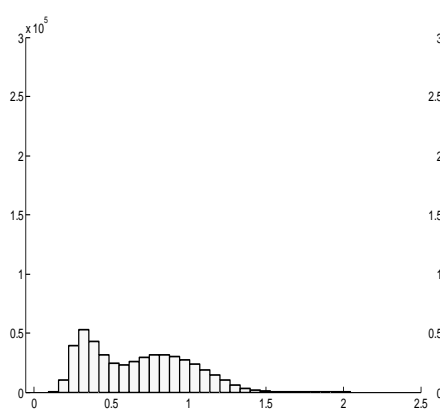

(a) Stat. of original PDSD

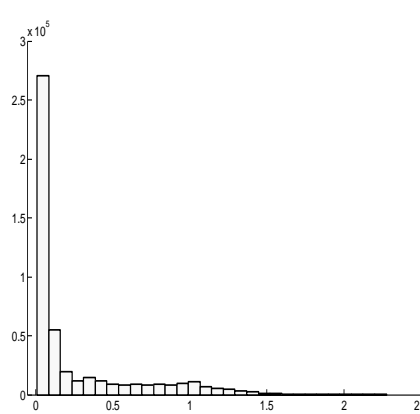

(c) Stat. of PDSD by Sigma filter Fig.4 Stat. of phase-derivative standard deviation over Bam, Iran

Tab.1 Comparison of the number of residues for three filters (Bam)

\begin{tabular}{|c|c|c|c|c|}
\hline & $\begin{array}{c}\text { Original } \\
\text { interfero- } \\
\text { gram }\end{array}$ & $\begin{array}{c}\text { The } \\
\text { interferogram } \\
\text { by wavelet } \\
\text { soft-threshold } \\
\text { filter }\end{array}$ & $\begin{array}{c}\text { The } \\
\text { interfero- } \\
\text { gram by } \\
\text { Sigma } \\
\text { filter }\end{array}$ & $\begin{array}{c}\text { The } \\
\text { interferogr } \\
\text {-am by } \\
\text { WSC filter }\end{array}$ \\
\hline $\begin{array}{c}\text { The number } \\
\text { of residues }\end{array}$ & 226910 & 59625 & 58148 & 45475 \\
\hline
\end{tabular}

According to fig.2, fig. 3 and fig.4, we can easily find that in two aspects of visualization effect and PDSD, the result of WSC filter is better than the results of wavelet soft-threshold filter and Sigma filter. The main reason is described as follows: Wavelet transform has good trait of time-frequency localization, but the effects of wavelet soft-threshold filter are related with the determination of thresholds. If the thresholds are too low, it will induce bad effects. Contrarily, larger thresholds are responsible for over-smoothness of image. Sigma filter can eliminate noise theoretically, but that is undesirable by experiments. Because of combining the advantages of wavelet transform and Sigma filter, WSC can not only remove the noises effectively, but also protect the edge information well.

We carry on the statistics of PDSD which correspond to the interferograms of three filters (see also Fig.4), and the number that lie in $[0,0.5]$ are the following: WSC filter 406,917, wavelet soft-threshold filter 397,395 and Sigma filter 388,752 . In the aspect of downsizing of the residuals (see tab.1): wavelet soft-threshold filter $73.72 \%$, Sigma filter $74.37 \%$ and WSC filter $79.96 \%$.

From above analysis and statistics, we know that the result of WSC filter is better than the results of Sigma filter and wavelet soft-threshold filter, while the results of Sigma filter and Wavelet soft-threshold filter are almost the same.

\section{CONCLUSIONS}

By analyzing the merits of wavelet transform and Sigma filter respectively, this paper proposes WSC filter and programmes to realize corresponding algorithm. To validate the proposed filter, one ASAR C-band interferogram over Bam, Iran (corresponding image block size is $600 \times 800$ pixels) is selected to carry on the experiment of filter and analysis. By selecting visualization effect of image, chart of PDSD and histogram of PDSD, number of residues as appraisal parameters, WSC filter is compared with Wavelet soft-threshold filter and Sigma filter.

From visualization effect of image, chart of PDSD and histogram of PDSD of InSAR interferogram, we know that WSC filter can eliminate noises more efficiently, compared with wavelet soft-threshold filter and Sigma filter. Besides, in the two aspects of PDSD standard deviation value $(<0.5)$ and residues decrement, the results are as following: WSC filter 406,917 and $79.96 \%$, wavelet soft-threshold filter 397,395 and $73.72 \%$, Sigma filter 388,752 and $74.37 \%$. The experiment proves that WSC filter is quite effective to eliminate InSAR interferogram noise.

\section{REFERENCES}

[1] Ferretti A., Claudio P., Fabio R. Multibaseline InSAR DEM Reconstruction: the Wavelet Approach [J]. IEEE Transactions on Geoscience and Remote Sensing, 1999, 37(2): 705-715.

[2] Massonnet D., Rossi M., Carmona C., et al. The Displacement Field of the Landers Earthquake Mapped by Radar Interferometry [J]. Nature, 1993, 364(8):138-142.

[3] Ding X. L., Liu G. X., Li Z. W., et al. Ground Subsidence Monitoring in Hong Kong with Satellite SAR Interferometry [J]. Photogrammetric Engineering and Remote Sensing, 2004, 70(10): 1151-1156.

[4] Eichel P. H., Ghiglia D. C. Spotlight SAR Interferometry for Terrain Elevation Mapping and Interferometric Change Detection [J]. Sandia National Labs. Tech. Report, 1993.

[5] Wang L. C., Wang Y. N., Mao L. P. An Algorithm of Interferometric Phase Filter of InSAR Based on Wavelet Analysis and Median Filter Algorithm [J]. Acta Geodaetica et Cartographica Sinica, 2005, 34(2): 108-112.

[6] Cai G. L., Liu G. X., Li Y. S. An Algorithm of Filtering InSAR Interferogram Based on Wavelet Phase Analysis [J]. Acta 
Geodaetica et Cartographica Sinica, 2008, 37(3): 293-300.

[7] Cai G. L., Li Y. S., Liu G. X. Wavelet-wiener Combined Filter and Its Application on InSAR Interferogram [J]. Journal of Remote Sensing, 2009, 13(1): 129-136.

[8] Mallat S. Theory from Multi-resolution Signal Decomposition: the Wavelet Representation [J]. IEEE Transactions on Pattern Analysis and Machine Intelligence, 1989, 11(7): 674-693.

[9] Carl T. The What, How and Why of Wavelet Shrinkage Denoising [J]. Computing in Science and Engineering, 2000, 2(3): 12-19.

[10] Vidakovic B. L., Ozoya C. B. On Time-dependent Wavelet denoising [J]. IEEE Transactions on Signal Processing, 1998, 46(9): 2549-2551.

[11] Lee J. S. Digital Image Smoothing and the Sigma Filter [J]. Computer Vision, Graphics and Image Processing, 1983, 24: 255-269.

[12] Ghiglia D. C., Pritt M. D. Two-Dimensional Phase Unwrapping Theory: Algorithms and Software [M]. John Wiley \& Sons, Inc, 1998: 70-82. 